\title{
A case of chorea-hyperglycemia-basal ganglia syndrome: a rare case of movement disorder
}

\begin{abstract}
Chorea hyperglycemia basal ganglia syndrome is a rare condition that manifests in setting of uncontrolled nonketotic diabetes mellitus. The objective of this case report is to highlight the importance of a physician to be aware of hyperglycemia as a cause of hemichorea/ hemiballismus which is referred to in medical literature as chorea-hyperglycemia-basal ganglia syndrome. ${ }^{1}$ This case involves a 60 years old Hypertensive and poorly controlled diabetic female who presented with choreiform movements of right upper and lower limbs for last one month. Her initial glucose level was $352 \mathrm{mg} / \mathrm{dl}$. CT scan of her brain showed no obvious abnormality except for hyper density left basal ganglia region. MRI brain (Plain+Contrast) revealed hyperintense lesion in left basal ganglia region; Left putaminal region showing signal changes likely metabolic in nature. With gradual control of her hyperglycemia, the movement disorder resolved. With exclusion of other causes of the movement disorder and resolution of her symptoms with control of blood sugar can lead to the inference that it was due to her uncontrolled diabetic status.
\end{abstract}

Keywords: chorea, hyperglycemia, basal ganglia syndrome, movement disorder
Volume 7 Issue 4 - 2020

\author{
Rupak Chatterjee,' Supratik Bhattacharyya ${ }^{2}$ \\ 'Post Graduate Trainee (PGT) MD Tropical Medicine, School of \\ Tropical Medicine, West Bengal University of Health Sciences \\ (WBUHS), India \\ ${ }^{2}$ Consultant Endocrinologist, AMRI, Salt Lake, India
}

\begin{abstract}
Correspondence: Dr Rupak Chatterjee, Post Graduate Trainee (PGT) MD Tropical Medicine, School of Tropical Medicine, West Bengal University of Health Sciences (WBUHS), West Bengal, India, Tel 6290I56807,

Email rupakchatterjee95@gmail.com
\end{abstract}

Received: September 0I, 2020 | Published: November 25, 2020

\section{Background}

Hyperglycemia induced involuntary movements are uncommon manifestations of diabetes seen especially in those with poor glycemic control. Of these, basal ganglia syndrome is a rare entity which can mislead the unwary. ${ }^{2}$ As the prevalence of Diabetes mellitus is increasing day by day; all physicians should be aware of uncontrolled hyperglycemia to be a cause of movement disorder. It is important for early diagnosis and management and also to avoid some expensive and cumbersome investigations.

\section{Case}

A 60yrs female K/C/O T2DM \& HTN (poorly controlled) presented with $\mathrm{H} / \mathrm{O}$ abnormal, non-purposeful, involuntary movements involving right upper and lower limbs for last 1 month. On examination, patient was alert, conscious, cooperative. Pallor, Cyanosis, Icterus, Clubbing, Edema were absent. Pulse- 80/min, regular in rhythm. B.P-160/100mm Hg. Chest-bilateral clear with air entry. CVS-S1, S2 audible; Abdomen-soft, no organomegaly.

\section{Neurological examination}

- Revealed Hyperkinetic, rapid, semi purposeful, dance like, nonstereotyped, involuntary movement of right upper and lower limbs, Right upper limb more than lower limb. Power-4/5 Right limbs. No other significant abnormality.

- On admission, CBG was $352 \mathrm{mg} / \mathrm{dl}$. Patient was started on-tab Amlodipine (10mg), tab Telmisartan (40mg); Tab Metformin $500 \mathrm{mg}$ twice daily after meal and Inj Insulin mixtard (30/70) started $20 \mathrm{U} \& 10 \mathrm{U} \mathrm{s} / \mathrm{c}$ before breakfast and before dinner.

- On day 2; notes revealed, previous day evening CBG-499 mg/ dl; Day2 morning-295 mg/dl; B.P-170/100 mmHg and abnormal movement persisted.

- Her investigations revealed: CBC-Hb-10g\%, TWBC-9,500/ $\mathrm{mm}^{3}$, Plt- 5.32 lakhs $/ \mathrm{mm}^{3}$; FBS-317mg/dl, Urea- $23 \mathrm{mg} / \mathrm{dl}$, Creatinine- 1.1 $\mathrm{mg} / \mathrm{dl}$; Lipid profile-TG-301 mg/dl; Tcholesterol-369 mg/dl and
$\mathrm{Na}+: 136.7 \mathrm{meq} / \mathrm{l} ; \mathrm{K}+: 3.51 \mathrm{meq} / \mathrm{l}$. Urine for ketone bodies sent (dipstick urine-negative for ketone body). Neurology Refer written. Tab Aspirin added. Tab LACOSAMIDE 100mg 1tab twice daily started. CT scans Brain-no significant abnormality noted.

- With continuing hyperglycemia, movement disorder was persisiting. Chest X-Ray (PA) and USG whole abdomen revealed no abnormality. Urine: ketone body nil. fT4, TSH-WNL.

- Neurologists reviewed the case and gave Impression of the case as Hemichorea ;Precipitant being Hyperglycemia .CT Scan Brain reported no obvious abnormality except for hyper density left basal ganglia region. They advised for MRI brain and told to continue Tab Lacosamide 100mg 1 tab and advised vigilant control of hyperglycemia.

- As she had persistently raised CBG; Regular insulin in dose of $6 \mathrm{U}$ before breakfast, 8Ubeforelunch, 6Ubeforedinner started and Inj Glargine $10 \mathrm{u} \mathrm{s} / \mathrm{c}$ at bedtime added. Psychiatry Refer was done. Tab Risperidone $0.5 \mathrm{mg}$ and Tab Clonazepam $0.5 \mathrm{mg}$ at bedtime were added.

- CBG STILL HIGH $\rightarrow$ (Morning) CBG->250mg/dl. Inj glargine $16 \mathrm{u} \mathrm{s} / \mathrm{c}$; Tablet metformin 500mg 2tab twice daily; T Linagliptin $5 \mathrm{mg} 1$ tab once before lunch \& Inj regular Insulin dose also increased. Strict diabetic diet ensured.

- With control of hyperglycemia \& with the medications, the movement disorder resolved.

\section{MRI brain $\mathrm{P}+\mathrm{C}$ report}

Figure 1 Generalised cerebral and cerebella atrophic changes are seen with ischemic leukoaraiosis. Left putaminal region is showing signal changes? metabolic in nature. Hyperintense lesion left basal ganglia region. Absence of other metabolic abnormalities \& systemic processes like lupus, hyperthyroidism and uremia apart from uncontrolled DM. No History suggestive of Rheumatic fever. Plus, resolution of her movement disorder with control of hyperglycemia; 
led us to conclusion that-Chorea was likely due to uncontrolled hyperglycemia.

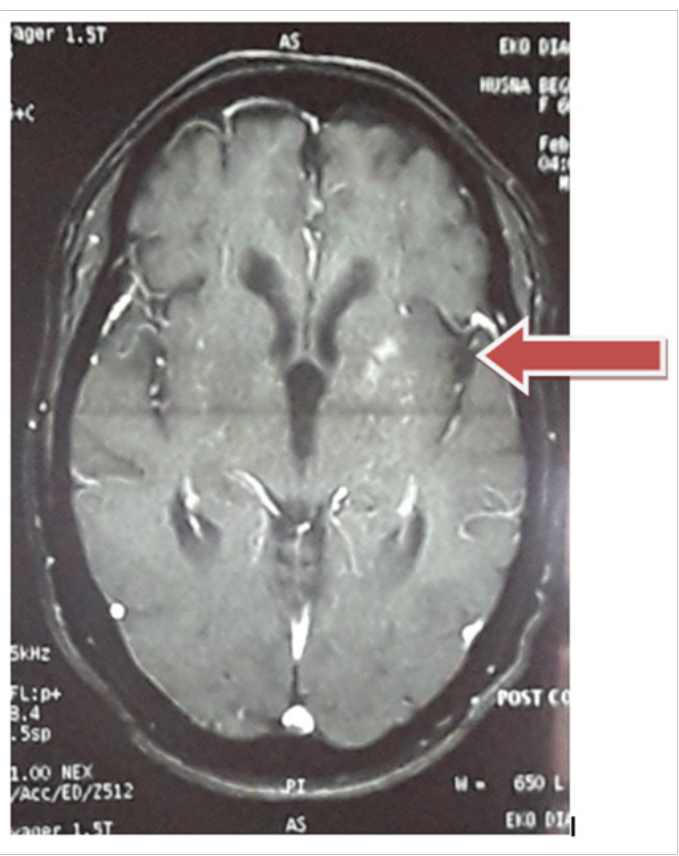

Figure I MRI Brain showing lesion in left basal ganglia.

\section{Discussion}

Chorea Hyperglycemia Basal Ganglia Syndrome is characterised by manifestation of hemichorea/hemiballismus with uncontrolled blood glucose levels. The exact etiology is not known but various hypotheses are there. According to some, hyperglycemia may impair cerebral auto regulation leading to hypoperfusion and anerobic metabolism. This results in depletion of GABA within neurons of basal ganglia. There is reduction in acetylcholine synthesis. ${ }^{3}$ As per another hypothesis, hyperglycemia induced hyper viscosity causes blood-brain barrier damage resulting in ischemia of striatal neurons. ${ }^{4}$
Patients reported are usually elderly with long standing Type 2 diabetes with poor control of blood glucose levels. ${ }^{1}$ Involuntary movements resolve with prompt normalisation of blood glucose levels. ${ }^{5}$ However, the radiological changes may take 2 to 12 months to disappear as per literature search. ${ }^{6}$ CHBG deserves awareness in light of the fact that once recognised and treated, it is a disorder of good prognostic value and so all clinicians-general physicians, endocrinologists and neurologists should be aware of it.

\section{Acknowledgments}

None.

\section{Conflicts of interest}

The authors declare that they have no conflicts of interests.

\section{Funding}

None.

\section{References}

1. Sperling M, Bhowansingh R. Chorea hyperglycemia basal ganglia syndrome in a 63 year-old male. BMJ Case Rep Med. 2018 :9101207.

2. Lietz TE, Huff JS. Hemiballismus as a presenting sign of hyperglycemia. Am J Emerg Med. 1995;13(6):647-648.

3. Cheema H, Federman D, Kam A. Hemichorea hemiballismus in nonketotic hyperglycemia. J Clin Neurosci. 2011;18(2):293-294.

4. Ahmad S, Mohan Babu P, Shenbagaraj L, et al. Rare case of choreahyperglycemia-basal ganglia (C-H-BG) syndrome. BMJ Case Rep. 2018:bcr2017223920

5. Padmanabhan S, Zagami AS, Poynten AM. A case of hemichoreahemiballismus due to nonketotic hyperglycemia. Diabetes Care .2013;36(4):e55-e56.

6. Suárez-Vega VM, Sánchez Almaraz C, Bernardo AI, et al. CT and MR unilateral brain features secondary to nonketotic hyperglycemia presenting as hemichorea-hemiballism. Case Rep Radiol. 2016:5727138. 